\title{
INFLUENCIA DE LA MOTIVACIÓN EN EL APRENDIZAJE MOTOR DE NIÑNOS CON PARÁLISIS CEREBRAL
}

\section{MOTIVATION INFLUENCE ON MOTOR LEARNING OF CHILDREN WITH CEREBRAL PALSY}

\section{Díaz B. Elisa1; Espinoza C. Angélica²; Parada T. Ana³; Zumelzu L. Yasna².}

\begin{abstract}
Resumen
La investigación tiene como principal objetivo determinar de qué manera influye la motivación durante el juego para la generación de aprendizaje motor de dos niños con parálisis cerebral en un Centro Educacional Integral de la ciudad de Valdivia. Bajo los fundamentos teóricos de la Ciencia de la Ocupación y la Terapia Ocupacional, se pretende identificar cómo la motivación influye en la conducta motora de los niños, favoreciendo o dificultando un aprendizaje motor durante el juego. La metodología utilizada es de carácter cualitativo, siendo su diseño el estudio de casos. Los principales resultados demuestran que para generar una motivación y para que ésta, a su vez, tenga una influencia en la generación de aprendizaje motor en estos niños, se requieren factores externos durante el desarrollo del juego, a saber: tipo de juego - particularmente juego propuesto y juego social-, intereses del niño y factores contextuales, los cuales repercuten en su desempeño ocupacional. Se concluye que el juego otorga herramientas para el aprendizaje motor, existiendo factores determinantes para que este pueda transformarse en una ocupación significativa. La motivación es clave para generar gran influencia en el aprendizaje motor de niños con parálisis cerebral, que impacta positivamente en el desempeño ocupacional, observándose mayor interacción con los pares, involucramiento, conductas motoras adaptativas y aumento de la participación durante el juego.
\end{abstract}

\section{Palabras Clave:}

Juego, Motivación, Aprendizaje Motor y Parálisis Cerebral.

\begin{abstract}
The main goal of this research is to determine the role of motivation in play regarding motor learning in two kids suffering from cerebral palsy in an Integral Educational Center in Valdivia. Under the theoretical basics of Occupational Sciences and Occupational Therapy, the idea is to identify how motivation influences the kids' motor behavior, making it easy or making it difficult to learn while playing. The methodology used has a qualitative nature, where its design is the study of different cases. The main results show that in order to generate a motivation, and for this motivation to have an influence on a motor learning in these kids, external factors are also required during the development of play, such as: types of play (particularly proposed play and social play), kid' interests, and contextual factors, which affect their occupational performance. In conclusion, play provides tools for motor learning. There are also critical factors for learning to become a significant occupation, where motivation is a key factor to generate a great influence on motor learning when it comes to kids affected by cerebral palsy. This impacts the occupational performance in a positive way, where a higher interaction with peers, involvement, adaptive motor behaviors and an increase of participation during play can be observed.
\end{abstract}

\section{Keywords:}

Game, Motivation, Motor Learning and Cerebral Palsy.

1 Licenciada en Ciencias de la Ocupación y Terapeuta Ocupacional. Escuela de Terapia Ocupacional, Facultad de Medicina de la Universidad Austral de Chile. Fono: 5663293510 Fax: 5663214475 Email: esctocupacional@uach.c

2 Licenciada en Ciencias de la Ocupación y Terapeuta Ocupacional. Escuela de Terapia Ocupacional, Facultad de Medicina de la Universidad Austral de Chile.

3 Licenciada en Ciencias de la Ocupación, candidata a Terapeuta Ocupacional. Escuela de Terapia Ocupacional, Facultad de Medicina de la Universidad Austral de Chile.

4 Licenciada en Ciencias de la Ocupación, candidata a Terapeuta Ocupacional. Escuela de Terapia Ocupacional, Facultad de Medicina de la Universidad Austral de Chile. 


\section{INTRODUCCIÓN}

Desde la perspectiva de la Terapia Ocupacional es importante tener en cuenta que su base es la ocupación, la cual se caracteriza por el valor y significado que la persona le otorga a sus ocupaciones, por lo que esta investigación se desarrolló utilizando la principal ocupación de un niño que es el juego. Éste se encuentra caracterizado por ser intrínseco, espontáneo, divertido, flexible, totalmente absorbente, vitalizante, desafiante, no literal, como un fin en sí mismo (Cohen, 1987; Ellis, 1973; Reilly, 1974; Rubin et al., 1983; Takata, 1971). En Terapia Ocupacional se utiliza el juego como una herramienta o medio facilitador del proceso terapéutico, siendo un vehículo práctico para captar la atención del niño, contribuyendo en el desarrollo de habilidades motoras específicas, perfeccionamiento sensorial, ejercicio de las capacidades perceptuales y cognoscitivas, sirviendo además como apoyo social, emocional y de desarrollo del lenguaje (Parham y Fazio, 1997).

Para que el niño se involucre durante el juego es de suma importancia que exista un grado de motivación. El concepto de motivación tiene variadas explicaciones, siendo entendida desde la psicología como una compleja integración de procesos psíquicos que efectúan la regulación inductora del comportamiento, pues determina la dirección, la intensidad y el sentido del comportamiento (González, 2008). A raíz de esto, Kielhofner (2004) plantea que la motivación surge de una interacción entre las características internas de la persona y los aspectos externos del ambiente para involucrarse en alguna ocupación, en relación a esto es de gran relevancia lograr determinar si la motivación es un factor influyente en la generación de aprendizaje motor durante el desarrollo del juego.

La neuroplasticidad es un facilitador de aprendizaje motor que se ha dirigido tradicionalmente al estudio de la adquisición o modificación de movimientos en individuos. Cuando existe una alteración en el desarrollo de habilidades, este aprendizaje permite la recuperación de las funciones y la readquisición de capacidades motoras perdidas por una lesión (Shumway-Cook \& Woollacott, sin fecha). Por lo tanto, el control motor es el cambio permanente del funcionamiento motor de un individuo causado como consecuencia de la práctica y es producido a través de la plasticidad cerebral, la cual se define como la reorganización de patrones distribuidos de la actividad cerebral, asociadas a las tareas normales que acompañan la acción, la percepción, cognición, y que además, compensan las funciones alteradas o perdidas como resultado de una enfermedad o lesión cerebral (Chávez, 2003).

Desde el control motor la motivación es un factor esencial que permite generar niveles altos de aprendizaje. "En el caso específico de la habilitación o rehabilitación de individuos con lesiones del sistema nervioso central, la motivación y concentración del paciente como también del tratante, son especialmente importantes, ya que pueden enfrentar el desafío" (Carrillo, 2005). Es por esto que cuando las personas se encuentran altamente motivadas dedicarán un mayor esfuerzo para la ejecución de una tarea o para generar una modificación en su realización, concientizándolo durante la práctica logrando estar dispuesto durante períodos más largos. En cambio aquellos que no presenten una motivación por aprender o ejecutar una práctica hacen solo la mitad del esfuerzo y desempeño necesario para alcanzar un cambio o nuevo aprendizaje (Schmidt, 2008).

Esta investigación surge a partir de la necesidad de generar conocimiento acerca de cómo la motivación influye en la generación de aprendizaje motor de niños y niñas con parálisis cerebral durante el juego. Si bien es cierto existe bibliografía que contextualiza estos conceptos, no se ha encontrado información que relacione la motivación, aprendizaje motor y parálisis cerebral como un todo, por lo cual se considera necesario entregar un estudio empírico que demuestre estos sustentos desde la Terapia Ocupacional, permitiendo entregar nuevos conocimientos a los profesionales que se desempeñen en áreas como la salud y la educación para brindar una intervención integral a los niños.

Bajo la mirada de la Terapia Ocupacional y la Ciencia de la Ocupación, la presente investigación tiene como objetivo principal determinar de qué manera influye la motivación en la generación de aprendizaje motor, durante el juego de dos niños con parálisis cerebral de un Centro Educacional Integral de la ciudad de Valdivia. Para el cumplimiento del mismo, se requiere identificar los factores que influyen en la motivación de un niño durante el juego, analizar cuáles inciden en la motivación de un niño durante el juego e identificar los cambios en el aprendizaje motor durante el proceso de tratamiento.

\section{Metodología}

La metodología que se utilizó en la investigación fue de tipo cualitativo, ya que propone una perspec- 
tiva holística de los sujetos, ya que busca describirlos en sus contextos; también se reconoce la influencia del investigador y se contextualiza la información generada (Hernández, Fernández \& Baptista, 2010). A partir de esto, se buscó descubrir una realidad particular, construida y mantenida de dos niños de ambos géneros con parálisis cerebral pertenecientes a un Centro Educacional Integral de la ciudad de Valdivia. El diseño de investigación utilizado fue el estudio de casos, que según Yin (citado en Sandoval, 2002, p. 92), "permite tratar exitosamente con una situación técnicamente distintiva en la cual hay muchas más variables de interés que datos observacionales". Para Stake (1998, p. 16) "es el estudio de la particularidad y de la complejidad de un caso singular, para llegar a comprender su actividad en circunstancias concretas".

En base a lo anterior, para el desarrollo de la investigación se seleccionaron dos casos de niños bajo los siguientes criterios de inclusión: niños que residan en la ciudad de Valdivia, entre diez y once años de edad, que posean parálisis cerebral con alto compromiso, ya sea motor como cognitivo; que realicen juego sensoriomotor y que pertenezcan al curso trastorno motor del establecimiento educacional integral escogido. Como particularidad de los casos seleccionados, se identifica que ambos niños, a pesar del alto compromiso motor que presentan en comparación con sus pares del centro educacional, logran participar e involucrarse en actividades dentro y fuera del aula. Además se destaca que la personalidad de ambos niños facilita la generación de un vínculo con terceros, lo cual permitió que los casos participaran de forma activa durante las intervenciones realizadas.

Para el presente estudio de casos, el levantamiento de la información se llevó a cabo a través de la observación participante, que permitió "Ilegar profundamente a la comprensión y explicación de la realidad por la cual el investigador participa de la situación obser- vada" (Wood, citado en Pérez, 2000 p, 41); notas de campo, que proporcionó la garantía de que la información adquirida no se perdiera y que ésta se encontrara disponible en todo momento (Pérez, 2010); entrevistas que permitieron obtener información sobre un tema o problemática de interés desde la perspectiva del entrevistado (Hernández, Fernández \& Baptista, 2010); grabaciones en video, puesto que Hopf menciona que las grabaciones proporcionan mayores opciones para una evaluación intersubjetiva de las interpretaciones (citado en Flick, 2004). Además, se aplicó de la escala de juego del preescolar de Susan Knox, que entregó una descripción del comportamiento desde el punto de vista del desarrollo del niño y el Test de jugueteo de Anita Bundy que permite evaluar la presencia de cuatro elementos: motivación, enmarcar, libertad para suspender la realidad y percepción del control. Otro recurso fue el Cuestionario Volicional Pediátrico que proporcionó ideas sobre la motivación del niño e información acerca del modo en que el medio ambiente sostiene o limita la volición del niño.

Considerando aquellos aspectos bioéticos fundamentales para la protección y derecho de los niños participantes en la presente investigación, es que se contó con el consentimiento informado otorgado por los padres y/o principales cuidadores responsables, así como también de la directora del Establecimiento Educacional. Cabe señalar que la investigación cuenta además, con la aprobación del comité de ética del Servicio de Salud de la ciudad de Valdivia.

La investigación fue realizada entre los meses de mayo y septiembre 20B? en el Centro Educacional Integral, donde se ejecutaron observaciones y aplicaciones de pautas de evaluación en aula y en los hogares de los niños, también se contó con la aplicación de seis entrevistas por cada caso (Tabla 1), realizadas a los familiares y profesionales del Centro Educacional Integral (Tabla 2).

Tabla 1

Caracterización de los Entrevistados.

\begin{tabular}{|c|c|c|c|}
\hline Caso & Instrumentos & Género & Relación con el niño \\
\hline \multirow{2}{*}{ Caso 1} & 6 entrevistas & 5 mujeres & 2 familiares \\
& & 1 hombre & 4 profesionales \\
\hline \multirow{2}{*}{ Caso 2} & 6 entrevistas & 5 mujeres & 2 familiares \\
& & 1 hombre & 4 profesionales \\
\hline
\end{tabular}


Tabla 2

Contenidos de entrevistas semi-estructuradas.

\begin{tabular}{|c|c|c|}
\hline Entrevistados & & Ejes Principales \\
\hline Profesionales & $\begin{array}{c}\text { Educadora diferencial del Centro } \\
\text { Educacional Integral } \\
\text { Terapeutas Ocupacionales del Centro } \\
\text { Educacional Integral } \\
\text { Estudiante en Práctica de Terapia } \\
\text { Ocupacional en CESFAM }\end{array}$ & $\begin{array}{l}\text { Tipo de Juego Interés } \\
\text { Conductas Motoras frente a un Juego de motivación } \\
\text { Participación de los Padres frente al proceso de Intervención } \\
\text { Observación de Progresos en el Componente Motor de los niños } \\
\text { Desempeño en Juego de los niños en distintos ambientes. }\end{array}$ \\
\hline Familia & $\begin{array}{l}\text { Padres de Caso } 1 \\
\text { Padres de Caso } 2\end{array}$ & $\begin{array}{c}\text { Observación de Progresos en el Componente Motor de los niños } \\
\text { Cambios observables en los niños en el desempeño durante el } \\
\text { juego Interés } \\
\text { Conductas Motoras frente a un Juego de motivación. }\end{array}$ \\
\hline
\end{tabular}

Como estrategia de validación de la información obtenida se utilizó la triangulación entendida como la estrategia que consiste en comprobar las inferencias extraídas a partir de una fuente de información, mediante el recurso de cada una de éstas (Hammerley \& Atkinson, 2005). Esta estrategia de validación nos permitió obtener datos de diferentes perspectivas en relación al concepto de motivación y aprendizaje motor en base a la utilización del juego como herramienta de intervención. Para describir el análisis de la información recopilada, se utilizó la narrativa, que permitió relatar las observaciones en los diferentes contextos, las diversas entrevistas realizadas, ya sea a los padres como a los profesionales que trabajan con los niños.

\section{Resultados}

Con el objetivo de conocer a los casos involucrados en la presente investigación, se realizó un perfil de las características de cada caso considerando género, edad, diagnóstico, topografía, tipo de juego y participación ocupacional (tabla 3).

Tabla 3

Caracterización de los Casos

\begin{tabular}{|c|c|c|}
\hline Caracterización & Caso 1 & Caso 2 \\
\hline Género & Masculino & 11 años \\
\hline Edad & 10 años & Parálisis Cerebral Mixta \\
\hline Diagnóstico & Parálisis Cerebral Espástica & Hemiparesia doble \\
\hline Topografía & Cuadriparesia & Sensoriomotor \\
\hline Tipo de Juego & Sensoriomotor & \\
\hline
\end{tabular}




\begin{tabular}{|c|c|c|}
\hline Caracterización & Caso 1 & Caso 2 \\
\hline Participación Ocupacional & Dependiente AVD Instrumentales & Dependiente en AVD (colabora en \\
& Asiste a Establecimiento Educacional \\
Integral de la ciudad de Valdivia. & higiene menor y en alimentación) \\
& Contexto social favorece & Asiste a Establecimiento Educacional \\
& & Integral de la ciudad de Valdivia. \\
\hline
\end{tabular}

Los principales hallazgos que se obtuvieron en esta investigación se dividieron en tres temas: juego libre, juego propuesto y juego social, a partir de los que se abordó la influencia de la motivación y el control motor en los niños; del mismo modo se obtuvieron resultados en relación al ambiente físico y social en el que éstos se desempeñan.

\section{Juego Libre}

\section{Influencia de la Motivación}

En relación a lo observado durante el juego libre, caso 1 presenta dificultades para involucrarse en diferentes actividades de su interés por el simple placer de hacerlas, logrando de manera ocasional permanecer en el juego. Al brindar un apoyo externo que le ofrezca una máxima estimulación, caso 1 logra manifestar curiosidad cuando existen elementos en el entorno que sean de su interés, incrementando así su nivel motivacional, lo cual se hace evidente, ya que el niño logra llevar a cabo ciertas acciones tales como tomar un objeto, manejarlo o botarlo.

En relación al caso 2, la niña logra permanecer involucrada cuando existen elementos de su interés en el ambiente sin necesidad de recibir apoyo de terceros, sin embargo cuando estos elementos no se encuentran presentes se observa que la niña no logra desarrollar juego libre, ya que la mayor parte del tiempo presenta dificultades para concentrarse en un objetivo específico y claro.

Se identifica que este tipo de juego exige una alta demanda de habilidades, por lo cual en ambos casos se observan dificultades para persistir en éste por un periodo de tiempo prolongado, abandonando o disminuyendo su participación activa.

\section{Control Motor}

Durante juego libre, caso 1 evidencia patrones de movimiento básicos y globales cuando los elementos presentes en el ambiente son de su interés, logrando realizar alcances con cierta dificultad. Se observan bajos niveles de atención durante el desarrollo del juego, lo cual se evidencia en que la mayor parte del tiempo el niño no dirige su mirada ni orienta su cuerpo hacia la manipulación de los distintos objetos presentes en la sala.

En relación al caso 2, durante el juego libre la niña logra patrones de movimientos básicos y globales cuando los elementos presentes en el ambiente son de su interés, logrando realizar alcances de objetos que atraen su atención, iniciando acciones de manera activa principalmente en actividades motoras gruesas, moviendo manos y pies sólo hacia estos elementos.

\section{Juego Propuesto}

\section{Influencia de la Motivación}

Se observa en el caso 1 que para iniciar acciones el niño requiere de instigación física y verbal, sin embargo de manera progresiva y a medida que se desarrollan las actividades de este tipo de juego, el niño logra permanecer involucrado en la actividad requiriendo de menor apoyo por parte de terceros. En cuanto a los parámetros atencionales logra atención sostenida durante la actividad, dependiendo de si ésta es de su interés, además muestra sus preferencias de manera espontánea, que se manifiesta por medio de gestos y conductas colaborativas en aquellas actividades, permaneciendo involucrado en éstas. El niño expresa placer con el logro, lo cual es observable mediante sus constantes sonrisas al momento en que realiza la tarea de forma efectiva, por ende continúa en ésta hasta completarla. 
En relación al caso 2, se observa que la niña en juegos propuestos que involucren sus intereses, inicia acciones de manera voluntaria hacia un objetivo específico sin requerir de estímulos externos, ya que realiza movimientos de forma espontánea intentando producir efectos o cambios en el ambiente, evidenciándose un alto nivel motivacional. Se observa que la niña se concentra en la tarea, no abandonándola ni frustrándose si ésta no es lograda; por el contrario, intenta resolver los problemas que se presentan para poder continuar. Busca espontáneamente desafíos que demanden mayores habilidades motoras y cognitivas de las que comúnmente utiliza, presentando la intención de enfrentar niveles de desempeño más alto y persistir en el objetivo sin abandonarlo, cuando el juego propuesto se basa en sus intereses.

\section{Control Motor}

En el caso 1, se observa que el niño logra realizar con dificultad las prensiones gruesas, siendo parcialmente efectiva la prensión cilíndrica y esférica; en cuanto a la ejecución de prensiones finas, se evidencia dificultad severa, ya que éstas no son logradas. A pesar de esto, cuando el niño se encuentra en posición prona logra apoyo asimétrico en codo, dirige su mirada y orienta su cuerpo hacia la manipulación de los distintos objetos presentes en la sala, siempre y cuando sean del interés del niño.

En relación al caso 2, la niña explora y practica habilidades motoras durante la tarea orientando su cuerpo hacia algo o alguien de manera voluntaria, sin requerir de apoyo adicional; además interactúa con los objetos tocándolos y manipulándolos. Se observa que la niña demuestra su inclinación o preferencia hacia el juego propuesto basado en sus intereses mediante expresiones faciales, movimientos (ej: sonrisa, juego de manos) y vocalización, intentando mantener un buen nivel de desempeño pese a sus dificultades principalmente motoras; por ejemplo logra desplazarse de un extremo hacia otro de la sala de juegos para dirigirse hacia un propósito determinado.

\section{Juego Social}

\section{Influencia de la Motivación}

Se observa en el caso 1, que el niño inicia acciones hacia otros por sí solo de manera ocasional, requiriendo de un apoyo constante para realizar este tipo de acciones, aun cuando se encuentra en un ambiente social favorable que conoce y en el que demuestra estar cómodo. El niño se concentra en las diferentes tareas durante este contexto y logra mostrar preferencias frente a ciertas actividades por sobre otras. Se observa que el caso 1 permanece involucrado durante el juego con sus pares, orientando su atención hacia ellos, siendo capaz de apoyar a sus compañeros principalmente a través de lenguaje corporal con sonrisas y gestos. Finalmente, intenta producir efectos en su ambiente social, resultando involucrado en este ítem, requiriendo de mínimo estímulo por parte de su ambiente para mostrar la conducta de intención.

En relación a la motivación en juego social, el caso 2 logra iniciar frecuentemente una nueva actividad con otros, expresando con moderada dificultad lo que necesita de manera verbal o no verbal. También se muestra espontánea al momento de expresar placer con el logro, una vez que se percibe efectiva en una actividad de interacción con otros demuestra orgullo de sí misma mediante la risa, vocalizando sonidos y permaneciendo en la misma actividad. La niña intenta producir efectos en su ambiente social, disfrutando mucho de aquellas actividades que generan reacciones positivas en otras personas, tales como risa o sorpresa; este tipo de comportamiento es realizado de manera voluntaria y continua.

\section{Control Motor}

En relación al caso 1, el niño intenta producir efectos en su ambiente con cierto grado de apoyo externo, además constantemente hace gestos con la intención de comunicarse con sus pares dirigiendo su mirada, orientando y maniobrando su cuerpo hacia ellos cuando éstos Ilaman su atención. 
En relación al caso 2, la niña logra entregar claves comprensibles verbales, faciales o corporales que indiquen cómo el otro debe actuar hacia ella y actúa acorde a las claves de juegos de otros. Presentando intención comunicativa a través de lenguaje no verbal, dirigiendo su cuerpo hacia otros, manifestando agrado o descontento a través de expresiones faciales y verbalmente por medio del balbuceo.

\section{Ambiente}

En cuanto a la dimensión social del contexto escolar, la Terapeuta Ocupacional del Centro Educacional Integral relata que "ambos niños tienen una orientación hacia actividades y juegos más sociales, disfrutando de la interacción con sus pares". Esto se evidenció mediante la observación realizada, ya que los niños participaban activamente de las actividades que requerían el involucrarse con un otro, generando una complicidad grupal y demostrando disfrute durante el juego.

En relación a la dimensión social del contexto familiar del caso 1, la madre refiere: "en la casa nosotros hacemos los ejercicios que nos han enseñado los tíos, pero en general no juego con él"; esto demuestra que pese a seguir las indicaciones otorgadas por los diferentes profesionales, no incentivan de manera constante la exploración del niño en su ambiente físico por medio del juego, disminuyendo su motivación e involucramiento en éste. En cuanto al caso 2, la Terapeuta Ocupacional de CEI destaca que "ella tiene un ambiente que le facilita que realice muchas cosas, que si va a comer que coma y que se ensucie, la mamá le ayuda cuando ve que la niña no puede". Lo anterior se evidenció durante la observación en el hogar de la niña, ya que la madre permitía la exploración de éste y de los diferentes elementos presentes.

En cuanto a la dimensión física del ambiente escolar, se observa que el CEl cuenta con luz natural y artificial lo que favorece la exploración por parte de los niños, además existen diversos materiales y amplios espacios que facilitan que participen y se involucren en diferentes juegos. En lo que respecta a la dimensión física del ambiente familiar, se pudo observar en ambos casos características similares, ya que estos hogares cuentan con espacios reducidos y pocos elementos que representen los intereses de los niños.

\section{DisCUSIÓN}

En relación a la influencia de la motivación, se logró establecer que durante las sesiones individuales existe un involucramiento activo por parte de los niños en actividades de su interés, evidenciándose en el aumento de la participación y demostrando disfrute a través de conductas comunicativas tales como sonrisas, contacto visual y balbuceos. Esto se logró observar en actividades que involucren la interacción social y que cuenten con la presencia de elementos sonoros, tecnológicos, como celulares y/o computadores. Asimismo, de acuerdo a lo observado se logró visualizar un aumento de la motivación en juegos propuestos por otros, alcanzando niveles de alerta acordes a la actividad, desarrollan permanencia durante el juego, evidenciando cambios posturales, principalmente en miembro superior y tronco.

En base a lo anterior, se observó durante la investigación que ambos niños, al inicio del juego propuesto, se involucran por medio de una motivación orientada a factores externos, a través de una actividad estructurada por un otro, que sea del interés del niño y motivándolo a participar en éste ya sea por medio de lenguaje verbal y no verbal. No obstante, a lo largo del juego y a través de la experiencia, los niños logran alcanzar una motivación inclinada a factores intrínsecos, transformándose este tipo de juego en una ocupación significativa, de su interés y voluntaria. Lo anterior se complementa con lo planteado por la autora Anita Bundy, quien establece que la motivación intrínseca se refiere a algún aspecto de la actividad en sí misma, en lugar de una recompensa externa, que proporciona el impulso para la participación de las personas en la actividad (citado en Parham y Fasio, 1997).

A partir de esto, se pudo reconocer que la motivación genera un cambio a nivel motor cuando la actividad o juego propuesto es del interés del niño, según refiere un estudiante en práctica profesional de Terapia Ocupacional del Centro Comunitario de Rehabilitación (CCR), se observaron avances en los aspectos motores, principalmente en "relación a control cefálico, control de tronco, movilidad de extremidades superiores, disminución de tono postural, extensión de ambas extremidades superiores, logrando alcances de objetos de poco peso". 
Por otro lado, lo relatado con anterioridad se refuerza en la investigación, ya que en actividades que no son del interés de los niños se produce una disminución en su nivel de participación, mostrando una actitud pasiva y de evitación, no se logra permanencia en la actividad e involucramiento activo, observándose principalmente durante el juego libre, ya que en éste se requiere de habilidades más complejas que involucren un mayor compromiso de funciones tales como la ideación, planificación y ejecución para llevar a cabo actividades, las que podrían encontrarse aún más interferidas debido al cuadro clínico de base.

En relación a lo expuesto anteriormente, Csikszentmihalyi (1990) plantea que cuando existe un equilibrio entre los desafíos de la actividad y las habilidades de las personas se logran estados de flujo, el cual se define como un estado subjetivo que las personas experimentan cuando están completamente involucradas en algo hasta el extremo de olvidarse del tiempo, la fatiga y de todo lo demás, excepto de la actividad en sí misma. En este caso, se observó que cuando los desafíos propuestos por las investigadoras en las actividades de juego eran acordes a las habilidades motoras que los niños poseen, lograron alcanzar estos estados de flujo, los cuales potenciaban su desempeño motor al máximo, favoreciendo un involucramiento efectivo durante el juego.

Por otra parte, cuando los desafíos durante el juego superaban las habilidades de ambos niños, éstos adquirían un rol pasivo observándose una disminución de los niveles de alerta, escasa interacción con los objetos, disminución de control cefálico y de tronco, los cuales se vieron reflejados en el bajo involucramiento que los niños desarrollaron frente a una actividad de juego libre, observándose menor motivación. De este modo, se argumenta que el flujo puede cumplir un papel importante en la evolución humana, en cómo las personas cuando experimentan un alto nivel de motivación, utilizan sus habilidades para enfrentar los desafíos del medio ambiente.

Durante el proceso de investigación se observó el desempeño de los niños dentro del contexto escolar y familiar, bajo perspectivas físicas y sociales, ante lo cual Kielhofner (2004) define el medio ambiente como las características físicas y sociales particulares de un contexto que tienen un impacto sobre lo que se hace y sobre el modo en que se hace, así cada medioambiente ofrece una cantidad de oportunidades, recursos, demandas y limitaciones que influyen en la realización de diversas ocupaciones y que tienen un impacto potencial sobre los niños.

En cuanto a la dimensión física y social del contexto escolar en el que se encuentran ambos niños, éste se caracteriza por poseer elementos de interés, espacios y apoyo social que entregan un ambiente enriquecido con mayores oportunidades y que potencian el desempeño.

En relación a la dimensión social del contexto familiar del caso 1, se observa que éste se encuentra en un ambiente que limita su participación no proporcionando las instancias de juego necesarias, impactando directamente en la motivación de éste. Lo anterior se contrapone con lo observado en el caso 2, ya que la niña se encuentra en un ambiente que la motiva a participar activamente en diversas actividades de juego que le proveen de experiencias y le entregan instancias que favorecen la adquisición de nuevas habilidades motoras, observándose a través de los avances en las diversas áreas de participación ocupacional de la niña a lo largo de su vida.

Esta investigación pretende establecer una base para próximos estudios en la línea de Ciencias de la Ocupación y Neurociencias en el área infanto-juvenil, abriendo una puerta escasamente explorada en el campo laboral, donde los Terapeutas Ocupacionales logren un abordaje integral, desde la premisa de que la motivación es un factor influyente en la generación de aprendizaje motor durante el juego, siendo considerado éste como la principal ocupación del niño.

Se plantearon objetivos dirigidos a fundamentar dicha premisa en relación al objetivo que pretende identificar los elementos que inciden en la motivación de un niño durante el juego, se logra destacar la importancia de los factores contextuales, los intereses, el juego propuesto y social, los cuales inciden en el aumento de los niveles motivacionales durante el desempeño de ambos niños. Por ende, la motivación es la llave o ingrediente clave para producir el conocimiento o un cambio (Schmidt, 2007), empíricamente este cambio se vio reflejado en el aprendizaje motor que los niños lograban al involucrarse de manera activa durante el juego.

En relación al objetivo que busca analizar los factores que inciden en la motivación de un niño durante el juego, los factores contextuales se pueden presentar como un facilitador en la motivación de los niños durante el juego, debido a que puede proporcionar oportunidades o instancias de estimulación en los más diversos entornos y otorgar posibilidades de interactuar con otros, ya sea con pares o familiares. Por otra parte el 
contexto se puede transformar en una barrera, ya que si éste no otorga las instancias o elementos de interés que motiven a los niños, estos no adquirirán la experiencia necesaria para desarrollar habilidades motoras, limitando su participación ocupacional durante el juego.

De tal manera las características particulares del contexto, ya sean físicas o sociales, pueden influir en su desempeño ocupacional y en la generación de aprendizaje motor en los niños.

Asimismo, en cuanto a los intereses que los niños presentan, es posible identificar que la motivación presenta grandes influencias en el aprendizaje motor de estos niños con parálisis cerebral, ya que si los juegos son de su interés, la motivación impactará de manera positiva en el desempeño ocupacional logrando mayor interacción con pares, involucramiento y participación durante el juego. En cuanto a esto es que se evidencia un mejor desempeño de los niños fuera del aula, requiriendo de apoyo constante por parte del profesional, quien debe focalizar las actividades en los intereses de ellos, facilitando de esta manera el desarrollo de conductas más elaboradas en términos cognitivos, sociales y motores.

En relación al involucramiento y aumento de niveles motivacionales de los niños, se evidencia que cuando participan en juegos que son estructurados y propuestos por un tercero, logran estados de flujos que permiten aumentar sus habilidades motoras, generando en el tiempo aprendizaje motor. Frente a aquellas actividades de juego libre se observa que existe un menor involucramiento por parte de los niños, ya que éstas exigen de habilidades más complejas y en consecuencia, reaccionan de manera pasiva disminuyendo su participación en este tipo de actividades.

Por lo tanto, se considera de gran relevancia que en los contextos escolares y familiares se incluyan trabajos que favorezcan la motivación en niños con este cuadro clínico, siendo de gran importancia el trabajo en base al juego desde la infancia, puesto que es la principal ocupación en esta etapa evolutiva, y evidencia cambios clave a nivel motor, especialmente durante los períodos críticos, propiciando un mejor pronóstico a futuro.

A partir del desarrollo de la investigación se identificaron ciertas limitaciones en relación al estudio de casos. En primera instancia éste se encontró susceptible a ciertos sesgos que involucraron tanto la selección de los niños como el contexto en el cual se desempeñaban, provocando que los resultados no puedan ser replicables a una población más significativa; se obtuvo entonces, sólo la realidad individual de dos niños con determinadas particularidades.

Por otra parte, dentro de las ventajas de la aplicación del estudio de casos, se logró identificar que a partir de experiencias y prácticas reales, se puede establecer un vínculo con el hacer y contribuir en la práctica de la Terapia Ocupacional en esta área de intervención. El hecho de que la investigación sea acotada a casos particulares también permite profundizar ampliamente en ambos casos y de esta forma analizar la información obtenida de manera integral. Es posible que a partir del análisis y discusión del tema abordado surjan direcciones para futuros estudios que tengan relación con las temáticas anteriormente mencionadas.

A partir de lo expuesto anteriormente, se logra validar el rol del Terapeuta Ocupacional como agente activo dentro del proceso de rehabilitación, ya que posee las herramientas necesarias para reconocer los intereses del niño, generando así la motivación de éste. Además, condiciona el ambiente de acuerdo a las necesidades particulares del niño, para estimular el aprendizaje motor por medio del juego y según lo expuesto en esta investigación, con principal énfasis en el juego propuesto, el cual es utilizado como una herramienta terapéutica a través de actividades que tengan directa relación con los intereses de los niños.

Por otra parte, es de gran relevancia dar continuidad a la investigación, ya que a partir de ésta surgen nuevas interrogantes, entre las que se encuentra la manera en que la propia personalidad del niño influirá en que éste genere motivación ante una actividad de juego determinada, es decir cómo el tipo de personalidad del niño impacta en el desarrollo del aprendizaje motor. Otro factor a indagar en futuras investigaciones se relaciona con los resultados que podría arrojar el realizar esta investigación con niños en edad preescolar, apelando a los períodos críticos que en esta etapa existen. Finalmente, es relevante que este estudio sea aplicado en niños que no posean un cuadro neurológico de base, para que de esta manera se puedan determinar las diferencias existentes con la hipótesis propuesta mediante un análisis comparativo. 
Figura 1

Factores que inciden en la motivación de los niños

A continuación, y a modo de conclusión, se esquematizan aquellos factores relevantes que inciden en el aumento de los niveles motivacionales de los niños, impactando en la generación de aprendizaje motor en los niños.

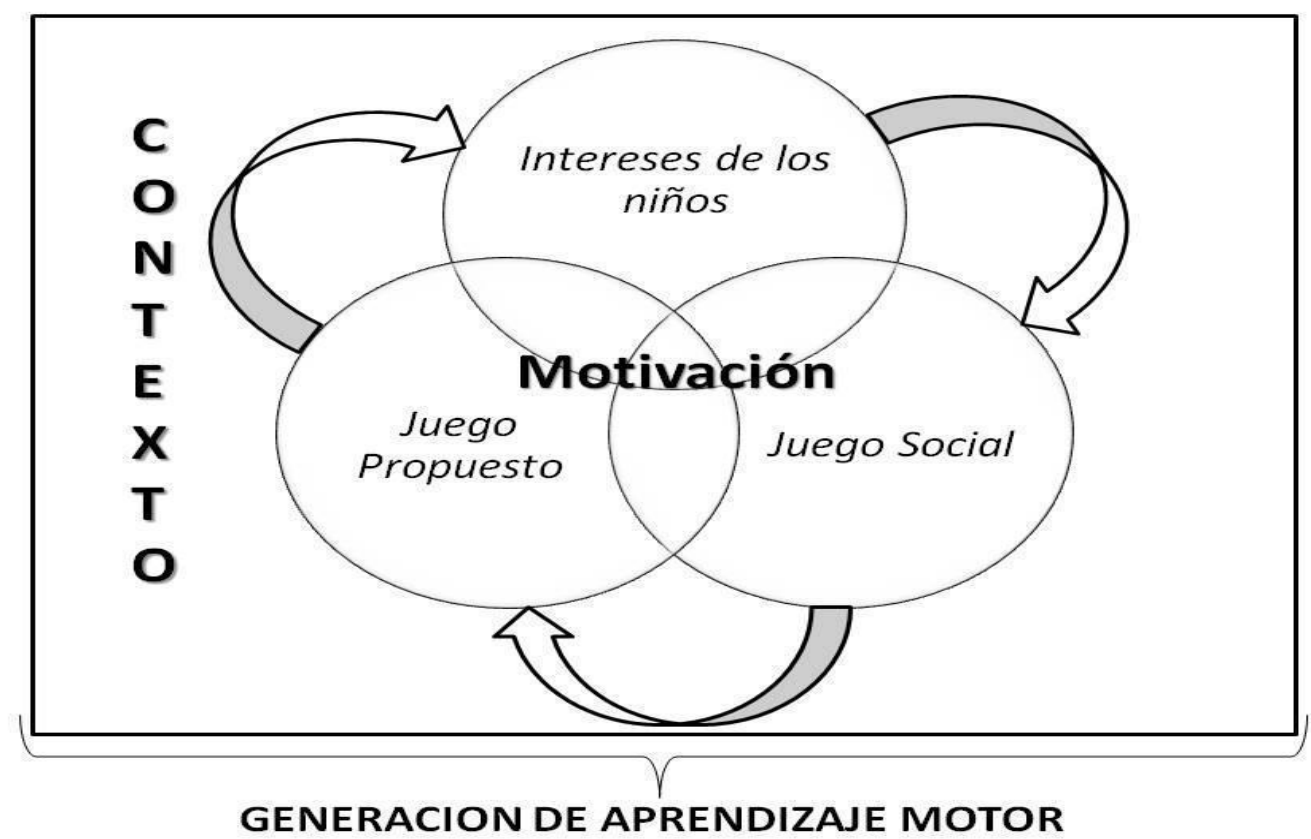

Figura elaborada por Díaz E.; Espinoza A.; Parada A.; Zumelzu Y.

\section{RefERENCIAS BibliográficAs}

Carrillo, M. (2005). "El aprendizaje motor en la práctica clínica: nuevos paradigmas en la rehabilitación de individuos con lesiones del sistema nervioso central .II parte". Kinesiología. Rev. Oficial del colegio de kinesiólogos de Chile. № 77. septiembre 2005, p: 17.

Chávez, R. (2003). "Neurodesarrollo Neonatal e Infantil: Un enfoque multi-inter y transdisciplinario en la prevención del daño" ( $1^{a}$ ed). Editorial Panamericana: México, D.F.

Csikszentmihalyi, Mihaly (1990). Flow: The Psychology of Optimal Experience. New York: Harper-Row.

Flick, U. (2004). "Introducción a la Investigación Cualitativa" (1 ed.). Madrid: Morata.

González, D. (2008). "Psicología de la motivación". Editorial Ciencias Médicas. México.

Hammersley, M. \& Atkinson, P. (2005). "Etnografía: Métodos de investigación" ( $\left.2^{\circ} \mathrm{ed}\right)$. España: Paidós.
Hernández, R., Fernández C. \& Baptista M. (2003). "Metodología de la Investigación". ( $3^{\circ}$ ed.). McGRA W- HILL: México.

Hernández, R., Fernández C. \& Baptista M. (2010). "Metodología de la Investigación". (5ed.). McGRA W- HILL: México.

Kielhofner, G. (2004). "Terapia Ocupacional: Modelo de Ocupación Humana" (3ed.). Editorial: Panamericana.

Parham, L. \& Fazio, L. (1997). "Play in occupational therapy for children". (1Ed.). St. Louis: Mosby, Inc.

Pérez, G. (2000). "Investigación cualitativa. Retos e interrogantes: II. Técnicas y Análisis". (3 ed.). Madrid: La Muralla.

Sandoval, C., (2002). "Investigación Cualitativa". ARFO Editores e Impresores Ltda.: Bogotá. Colombia.

Schmidt R. \& Wrisberg C. (2008): "Motor learning and performance". (4 Ed.).

Shumway-Cook, A, \& Woollacott M., (s.f.). "Motor Control". (3 Ed.)

Stake (1998). "Investigación con estudio de casos". (2 Ed.). Madrid: España 